\title{
Planificación de los servicios de epidemiología en los hospitales
}

\section{Planning of epidemiology services in hospitals}

\section{Planejamento de serviços de epidemiologia em hospitais}

María A. Quijije-Ortega ${ }^{\text {I }}$

maria.quijijeo@uleam.edu.ec

Miryam P. Loor-Vega

miryam.loorv@uleam.edu.ec

Alba A. Sornoza-Pin ${ }^{\text {III }}$

alba.sornozap@uleam.edu.ec

Viviana del Rocío Bacusoy-Mero

IV

vivibacusoy@gmail.com

Karol D. Castillo-Chávez

karol.castilloch@uleam.edu.ec

Gabriela J. Garcés-Vera

jggv_92@hotmail.com

Recibido: 27 de octubre de 2018 * Corregido: 18 de noviembre de $2018 *$ Aceptado: 10 diciembre 2018

I. Magister Gerencia en salud para el desarrollo local; Licenciada en Ciencias de la Enfermería; Docente de la Universidad Laica Eloy Alfaro de Manabí, Manta, Ecuador.

II. Magister en Gerencia en Salud para el Desarrollo local; Licenciada en Ciencias de la Enfermería; Docente de la Universidad Laica Eloy Alfaro de Manabí, Manta, Ecuador.

III. Maestría en Gerencia Salud Sexual y reproductiva; Licenciada en ciencias de la Enfermería; Docente de la Universidad Laica Eloy Alfaro de Manabí, Manta, Ecuador.

IV. Licenciada en Enfermería; Ministerio Salud Pública, Portoviejo, Ecuador.

V. Magíster en Gerencia Educativa; Docente de la Facultad de Enfermería de la Universidad Laica Eloy Alfaro de Manabí; Manta, Ecuador.

VI. Médico General; Hospital Rodríguez Zambrano, Manta, Ecuador. 


\section{Resumen}

En las últimas décadas se han producido importantes cambios en los servicios de salud, ocurriendo al mismo tiempo la cobertura universal de las prestaciones asistenciales y un enorme crecimiento en el número y coste de las tecnologías sanitarias. Por otra parte, los cambios en la sociedad afectan también a la configuración y transformación de los servicios de salud, que se tienen que ir adaptando a una población más educada y exigente, al mismo tiempo que demográficamente más envejecida. Todo ello ha ido llevando a un panorama complejo en la gestión sanitaria, desde la necesidad de contener el crecimiento del gasto sanitario, hasta la de manejar cantidades ingentes de información, pasando por la implicación de múltiples actores en la toma de decisiones, que irían desde el ciudadano de a pie, hasta el gestor político. En este sentido, las tendencias actuales se orientan a una mayor implicación de los usuarios y pacientes en los procesos asistenciales y de planificación.

La epidemiología puede considerarse a un tiempo disciplina científica y herramienta útil para ayudar a la toma de decisiones en todos los ámbitos de la gestión. Su papel se centra en aportar información fiable, conocimiento y previsión relevante para introducir racionalidad en la toma de decisiones. Esto es aplicable al entorno más macro de la gestión, es decir al proceso político y planificador (políticas de salud y salud pública), al de la meso gestión (cuyo ámbito de decisión afectaría a los centros e instituciones), y al nivel micro, que se correspondería con la gestión clínica, es decir con las decisiones que afectan directamente a los individuos de forma aislada o colectiva. Es quizás en este último ámbito, con el fenómeno de la medicina basada en la evidencia, donde la intersección entre epidemiología y gestión ha sido más amplia, por lo que queda mucho más camino por recorrer en otros ámbitos.

Palabras claves: Tecnologías Sanitarias; Gestión Sanitaria; Epidemiología. 


\section{Abstract}

In recent decades there have been major changes in health services, occurring at the same time the universal coverage of health care benefits and tremendous growth in the number and cost of health technology. On the other hand, changes in society also affect the configuration and transformation of the health services, that need to adapt to a more educated and demanding, at the same time as population demographically more aged. All this has been performing a complex panorama in health management, from the need to contain the growth of health spending, to handle huge amounts of information, through the involvement of multiple actors in the decision-making process, that would go from the citizen of a foot, until the political Manager. In this regard, current trends are oriented to a greater involvement of users and patients in health care processes and planning.

Epidemiology may consider both scientific discipline and useful tool to aid decision-making in all areas of management. His role focuses on providing reliable information, knowledge and relevant forecast to introduce rationality in decision making. This is applicable to the environment more macro management, namely the political process and Planner (public health and health policies), to the of management (whose scope of decision will affect centres and institutions), meso it and micro level, which corresponds with the clinical management, i.e. with decisions that directly affect individuals in isolated or collectively. It is perhaps in this last field, the phenomenon of medicine based on the evidence, where the intersection of epidemiology and management has been wider, by what is much more way to go elsewhere.

Key words: Health Technology; Health Management; Epidemiology. 


\section{Introducción.}

Los gobiernos de los países de la Región tienen la responsabilidad de comprender el proceso permanente de mudanza social, económica y política que afecta a América Latina y el Caribe y de facilitar las acciones del sector salud y de la sociedad como un todo para permitir, con eficiencia y equidad, la solución de los problemas prevalecientes y el alcance de la meta de salud para todos en el año 2000 que todos los gobiernos del mundo, y en especial los de las Américas, se han propuesto. Las tareas de formar conocimiento y de emplearlo adecuadamente son parte de la inteligencia que se requiere para la monitoria y explicación de la situación en que vivimos. A este fin, los enfoques conceptuales y métodos lógicos de la epidemiología tienen un papel extraordinario que cumplir no solo en la dimensión útil y necesaria de la vigilancia y como instrumento esencial para el control de enfermedades, sino sobre todo en la dimensión amplia de la comprensión integral de la salud y de sus prácticas. Con base en esta perspectiva, desde 1983 se han vitalizado los esfuerzos nacionales para promover el dominio y la utilización del enfoque epidemiológico como elemento esencial en la transformación de los sistemas de salud hacia el logro de sus elevados fines. En lo que concierne al desarrollo de los recursos humanos en salud, ha habido avances tangibles en la formación académica regular en epidemiología, así como intentos para remediar su carencia a nivel de los trabajadores incorporados en los sistemas de salud. No obstante, al persistir la separación entre la teoría y la práctica, los esfuerzos de las instituciones educativas y de las instituciones de servicio no han alcanzado el desarrollo que exige la complejidad del espectro de necesidades e ideales de salud de la sociedad actual. La urgencia y naturaleza de las acciones y transformaciones requeridas así como la gran rotatividad del personal de los niveles de decisión abren un espacio para la respuesta coyuntural apropiada al contexto, a las políticas sectoriales y a las funciones y necesidades de los recursos humanos. (1)

\footnotetext{
64 Vol. 5, núm. 1, enero 2019, pp. 3-31 María A. Quijije-Ortega; Miryam P. Loor-Vega; Alba A. Sornoza-Pin; Viviana del Rocío BacusoyMero; Karol D. Castillo-Chávez; Gabriela J. Garcés-Vera
} 
Este esfuerzo complementa la formación regular y facilita una interacción mayor de ambos procesos que es mutuamente beneficiosa.

Por esta razón, hemos creído importante elaborar para su difusión una síntesis de algunas de las experiencias nacionales sobre capacitación en y para el servicio en epidemiología. Entendemos como tal, aquella que pretende preparar para la práctica o actualizar a los trabajadores de los servicios de salud en la adecuada ejecución de las funciones esencialmente ^epidemiológicas o de las funciones generales del sistema con componente epidemiológico.

La Epidemiología tiene la capacidad de convertirse en instrumento para la planificación y conducción estratégica de los sistemas de salud. Esto resulta útil a una gran diversidad de profesionales del sector y no sólo a los epidemiólogos; ya que le permite una visión sistémica y holística de la situación de salud de la población, y consecuentemente una mejor efectividad de las intervenciones sanitarias.

\section{Desarrollo.}

\section{Epidemiología}

La mayoría de las IH son de carácter endémico, es decir que se presentan de forma esperada tanto en sus características como en frecuencia. Ocasionalmente aparecen brotes o epidemias que se localizan en áreas específicas del hospital y están causadas por microorganismos particulares o con resistencia antimicrobiana inusual. (2) La incidencia es difícil de establecer porque estará en gran parte determinada por las características del nosocomio (estructura edilicia, tamaño, número de camas y servicios, tipos de servicios) y las medidas de control aplicadas. En general varían entre 2 y 25\% de los pacientes admitidos, correspondiendo las tasas más altas en servicios como los de

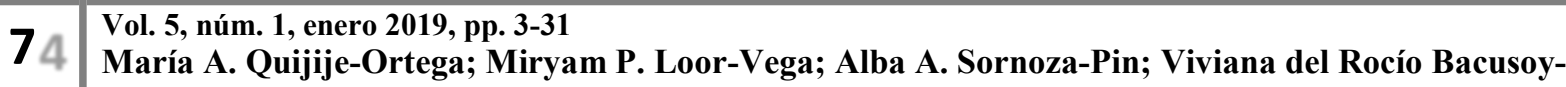
Mero; Karol D. Castillo-Chávez; Gabriela J. Garcés-Vera
} 
oncología, trasplantes, CTI, cirugía, y las más bajas a los servicios médicos, obstetricia y pediatría. Los agentes etiológicos de las $\mathrm{IH}$ incluyen bacterias, virus, hongos y parásitos, en ese orden de frecuencia. (3)

Epidemiología: Ciencia que trata del estudio de la distribución de las enfermedades, de sus causas y de los determinantes de su frecuencia en el hombre, así como del conocimiento de la historia natural en las enfermedades y del conocimiento de datos para una intervención orientada al control o erradicación de ellas. Su práctica se realiza a través del "método epidemiológico", basado en la observación de los fenómenos, la elaboración de hipótesis, el estudio o experimentación de éstas y la verificación de los resultados. Se ha dicho que es "una ciencia de lo poblacional o colectivo (como la sociología) pero construida del modo de las ciencias biológicas o naturales”(4).

En éste sentido, se describen tres etapas o fases del método epidemiológico:

1) Descriptivo: simple descripción de la distribución de los problemas o eventos de salud en términos de frecuencia en diferentes poblaciones o en diferentes grupos de una misma población.

2) Analítico: estudios diseñados especialmente para examinar la validez de las hipótesis formuladas. Esta validez puede tener un comienzo de apoyo en nuevos estudios descriptivos, en los cuales se seleccionan los grupos a comparar con base en su conocida mayor o menor exposición al posible factor causal.

3) Experimental: estudios de manipulación (producción, aplicación, supresión, modificación de frecuencia o intensidad) de la supuesta causa y observación ulterior de los resultados que tal manipulación determina sobre el supuesto efecto. En la práctica de la investigación, en la de los servicios locales de salud y en la de las 
instituciones sanitarias, la epidemiología ha centrado su interés en ciertos eventos o problemas de salud que, por diversas razones han surgido como prioritarios. De este modo se ha desarrollado la epidemiología de las enfermedades infecciosas, la de los accidentes, la de las enfermedades mentales, entre otras, y más recientemente, la epidemiología ambiental.

En relación entre poblaciones y la atención de los pacientes, se cubren temas como: medición de la frecuencia de las enfermedades, descripción de los patrones de incidencia de las enfermedades, evaluación de la utilidad de las pruebas diagnósticas, pruebas de la efectividad de los tratamientos, identificación de las causas de enfermedades, predicción del pronóstico de las enfermedades, toma de 2 decisiones relativa a estrategias terapéuticas, resumen de información disponibles sobre cuestiones clínicas.

En relación con el sistema de servicios, la misión central de la epidemiología es producir el conocimiento que permita profundizar la explicación de los procesos de salud (enfermedad), para facilitar la toma de decisiones relacionadas con la formulación de políticas de salud, la organización del sistema y las intervenciones destinadas a dar soluciones a problemas específicos. En función de esa misión, la investigación en epidemiología tendría que estar orientada a:

- Identificar, describir e interpretar los procesos que en el ámbito individual y colectivo, determinan la frecuencia y distribución de los problemas de salud.

- Facilitar una visión crítica de la situación de salud. - Contribuir para establecer la jerarquía de prioridades de los grupos de población y los determinantes de sus problemas.

- Seleccionar estrategias de intervención y evaluar su impacto. En cuanto la descripción y la explicación son actos de conocimiento o actos de investigación, las decisiones sobre la 
utilización de dichos conocimientos para la transformación de las condiciones de salud, constituyen actos políticos que involucran el conocimiento, la gobernabilidad del sistema, la capacidad de administrar, los recursos técnicos y financieros y el espacio de poder disponibles, en el marco de los proyectos e intereses de los diversos actores sociales.

La producción de conocimiento sobre la situación de salud y sus determinantes, debe articularse estrechamente con los procesos de decisión sobre prioridades a asignación de recursos.

Se identifican cuatro grandes campos de acción de la epidemiología en los sistemas de servicios

- Estudio de situación de salud en diferentes grupos de población, sus determinantes y tendencias.

- Vigilancia epidemiológica de enfermedades y otros problemas de salud.

- Evaluación del impacto en salud de los servicios y otras acciones sobre las personas, medio ambiente y condiciones de vida, y la evaluación de tecnología en función de su seguridad e impacto.

Los estudios de la situación de salud no pueden quedar restringidos a la enumeración de las principales categorías de la mortalidad y morbilidad, con algunos indicadores demográficos. Al formar parte del proceso de identificación y explicación de problemas prioritarios en función de su transformación deben orientarse a la evaluación sistemática de los problemas en salud y sus procesos determinantes, con la finalidad de informar la adopción de las decisiones y acciones requeridas para reducir el riesgo de enfermar y morir.

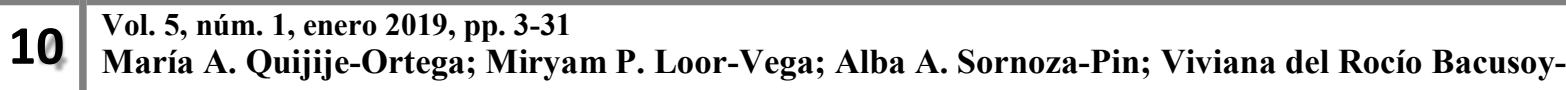
Mero; Karol D. Castillo-Chávez; Gabriela J. Garcés-Vera
} 
Las relaciones entre la Epidemiología y la Planificación (6)

La aplicación de la Epidemiología en la Planificación de Servicios de basa en la medición de las necesidades de la población a través del conocimiento de la demanda. De esta manera se utilizan los indicadores de demanda como expresión de las personas que reciben atención o que desean recibirla.

Este indicador expresa la necesidad cuando los servicios se distribuyen con equidad, en forma homogénea, para toda la población, y ésta posee una semejante accesibilidad a dichos servicios siendo, por lo demás aceptablemente satisfactorios los registros de las prestaciones médicas. Cuando ello no es así, el perfil de la salud puede ser distorsionado; tal es el caso cuando la demanda resulta un producto de la oferta o cuando se produce un sesgo contra los niveles socioeconómicos más descendidos. Por ello no se puede tomar la demanda de la atención como indicador de morbilidad y, en consecuencia, de necesidad: puede ser engañosa.

Al intentar determinar las necesidades sobre la base de la demanda, ésta puede resultar estimulada por un aumento de la oferta. En este caso, aquella debería ser regulada por la organización de ésta. También aquí la Epidemiología debería investigar la medida de las necesidades reales.

En cada etapa de la Planificación, los hechos deberían ser investigados e interpretados con metodología y criterio epidemiológico para la toma de decisiones. De esta manera, durante las etapas de diagnóstico, decisión y evaluación, la epidemiología será responsable de planear las necesidades, estimar los daños, valorar la importancia relativa de los fenómenos y determinar las opciones que existan para el curso de las acciones posibles. Valorando por último, el resultado de las 
mismas. Así se llegará a la toma de decisiones con la presentación del problema racionalmente estudiado y priorizado de acuerdo con las posibles opciones en términos de costo-beneficio.

Planificación y administración de los servicios de Salud (7)

Estas dan lugar al análisis de los aportes de la epidemiología a la administración de los servicios de salud a través de los diferentes pasos de la planificación.

Paso 1 "Diagnóstico"

Identificación de necesidades y problemas

El proceso comienza con la identificación de la necesidad de servicios de salud por parte de una población. Según Donabedian sugiere, esta necesidad puede ser vista cuando menos desde dos perspectivas: la del cliente (o paciente) y la del profesional. Como determinar las necesidades. Los enfoques varían en complejidad, costo, tiempo y efectividad. Son tres las funciones comunes a todos los enfoques: compilación (recopilación de datos a partir de fuentes existentes), desarrollo (producción de nueva información) e integración (síntesis de la información que se origina dentro y fuera de los límites del sistema).

Enfoque por indicadores. Existen tres indicadores dentro de esta clasificación:

- De salud: Resultan del análisis de los datos de morbilidad, mortalidad y, más recientemente, discapacidad. Entre las fuentes de esta información se encuentran los informes hospitalarios de internación y altas, las estadísticas confiables de enfermedades, las estadísticas de mortalidad materna, neonatal e infantil y las tablas de expectativa de vida e índices de discapacidad para poblaciones específicas. 
- Sociales: Son relevantes para identificar las necesidades de salud porque se correlacionan con la utilización de la atención. Las fuentes de información para la formación de indicadores sociales incluyen las estadísticas por edad, sexo, educación, antecedentes étnicos, vivienda, trabajo y consumo de alimentos.

- Extrapolación / suposición: Este método aplica los datos epidemiológicos a la prevalencia e incidencia de enfermedades y ciertas condiciones sanitarias en una pequeña población de referencia. El propósito es estimar las necesidades de salud asociadas con esas mismas condiciones en una población mayor. Enfoque por encuestas.

\section{Hay cuatro elementos:}

- Análisis de la utilización: Examina las necesidades en términos de la demanda de servicios. La demanda se mide por tipo y calidad de servicios efectivamente utilizados.

- Porcentaje en tratamiento: Analiza específicamente la utilización de servicios.

- Infraestructura de servicio y mano de obra: Se basa en la suposición de que los individuos que reciben atención realmente la necesitan.

- Encuestas tipo de la población general: Determinan las necesidades reuniendo datos de problemas.

La contribución de la epidemiología se centra básicamente en expresar los objetivos de manera cuantitativa y usando las relaciones de riesgo se puede disponer de factibilidad de reducir la incidencia o prevalencia.

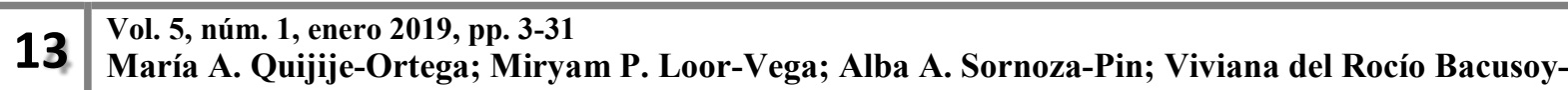
Mero; Karol D. Castillo-Chávez; Gabriela J. Garcés-Vera
} 


\section{Programación}

\section{Actividades para el logro de objetivos}

El marco que adopte el administrador resulta crucial para la generación de actividades y servicios aplicando un análisis costo beneficio.

\section{“Ejecución”:}

Movilización, coordinación de recursos. Aquí es donde operan y se ponen de manifiesto la mayoría de las funciones de administración. Es la prestación efectiva de servicios.

\section{“Evaluación”:}

Tres áreas:

- Financiera: trata de la contabilización de los costos.

- De procesos: Actividad de los programas.

- De resultados: Mide los efectos del programa para determinar si ha habido un cambio en el estado de salud como resultado del esfuerzo.

Es preciso que todas las organizaciones conozcan y entiendan la epidemiología de las enfermedades y su relación con la utilización de los servicios. Si deseamos mejorar el nivel de salud de la población de nuestro país, es necesario establecer un reordenamiento de las actuales prioridades de la atención de la salud poniendo mayor énfasis en la prevención de las enfermedades y en la promoción de la salud.

\footnotetext{
Mero; Karol D. Castillo-Chávez; Gabriela J. Garcés-Vera
} 
Los administradores de salud deben extender sus servicios a la comunidad de manera de atender no solo a los pacientes que se acercan a la consulta sino también a aquellos en situación de riesgo en virtud del medio ambiente, el estilo de vida, o la herencia (biológica).

El objetivo de los administradores de salud debe ser la salud de la comunidad en su totalidad y de sus miembros individualmente ofreciéndoles programas preventivos. Es necesario ampliar el mercado de promoción de la salud, de manera que la gente comience a hacerse más responsable del cuidado de su propia salud. De acuerdo con las actitudes que la gente asume frente a la vida, ciertos hechos (muerte, lesión, enfermedad) solo le ocurrían a otro, en cualquier momento de la vida y por casualidad. La realidad es que las probabilidades de que estos hechos ocurran a todos y a cada uno de nosotros son altas y que además es posible predecir perfectamente en qué etapa de la vida pueden ocurrir.

Aplicación en la administración de salud (8)

La epidemiología puede y debe ser utilizada a los efectos de la administración de los servicios de salud. Contribuye a la elaboración del diagnóstico de una comunidad respecto de la exigencia, naturaleza y distribución de la salud y la enfermedad. Constituye un medio para controlar la salud de la población. Más aún, utilizando los datos causales obtenidos por los epidemiólogos clásicos, los administradores pueden identificar individuos en situación de riesgo, su mercado o población objetivo potencial.

La epidemiología provee muchos de los elementos para la administración y planificación de los servicios de salud, y para su evaluación.

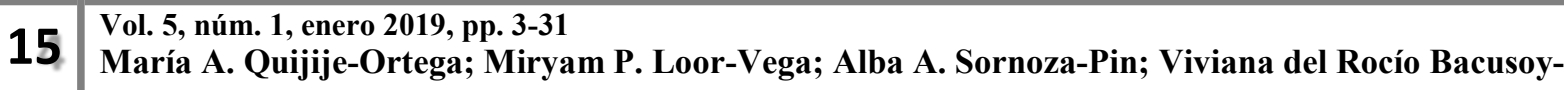
Mero; Karol D. Castillo-Chávez; Gabriela J. Garcés-Vera
} 
Administración: Proceso mediante el cual se supervisa la producción de los servicios (en este caso el de salud) o Funciones:

1. Planificación: Decidir anticipadamente lo que se desea hacer, lograr un conjunto de acciones coherentes para alcanzar ciertas metas.

2. Organización: Forma de relacionar personas y cosas de manera tal que se combinen en una unidad tendiente a un logro de los objetivos.

3. Dirección: imparte órdenes, supervisa, conduce, motiva y comunica.

4. Coordinación: Reúne, sincroniza personas y actividades para que funcione armoniosamente en el logro de los objetivos.

5. Control: Evalúa y corrige acciones de una organización para asegurar el logro de los objetivos. De la unión de estas funciones surge la toma de decisiones sobre la base de información.

Esta información puede ser "dura" (formal) o "blanda" (más o menos subjetiva). En cualquier caso los administradores procesaran algún tipo de información sobre la cual basaran sus decisiones que a su vez han de traducirse en acción organizada. La función de la epidemiología relativa a la administración de los servicios de la salud es proporcionar esta información "dura" para que constituya una base para la toma de decisiones.

- Planificación: es un proceso que se ocupa de reunir información utilizarla para el desarrollo y elaboración de las acciones y actividades de la organización. A las instituciones relacionadas con la salud, la epidemiología les brinda un método, dentro del proceso de planificación, para reunir información y establecer lineamientos para ejecutar las actividades o programas. o Tres son los niveles de planificación:

\footnotetext{
16 Vol. 5, núm. 1, enero 2019, pp. 3-31 María A. Quijije-Ortega; Miryam P. Loor-Vega; Alba A. Sornoza-Pin; Viviana del Rocío BacusoyMero; Karol D. Castillo-Chávez; Gabriela J. Garcés-Vera
} 
- Normativo: Se definen normas y procedimientos. Se planifica a corto plazo ante certeza y riesgo.

- Estratégico: Se establecen políticas, objetivos, metas, teniendo en cuenta los recursos disponibles y los aspectos que conforman el medio ambiente. Son a largo plazo.

- Tácito: Se definen responsabilidades, programas, presupuestos. Se planea a mediano plazo.

Es imposible realizar la planificación en forma aislada, por el contrario, siempre se deben tener en cuenta los valores sociales y el paradigma predominante o marco de salud que la afecta Las normas y objetivos propuestos dependen solamente de la posibilidad tecnológica de lograr algo. Gestión es la ejecución de lo planeado y producir resultados concretos.

Epidemiología de la utilización de los Servicios de Salud (9)

Factores determinantes de la utilización de los Servicios.

La epidemiología es la ciencia que estudia la ocurrencia, distribución y factores determinantes de la salud y la enfermedad en una población.

La utilización de los servicios de salud es una interacción entre consumidores y profesionales, donde se encuentran una amplia variedad de factores sociales y culturales.

\section{Factores Socioculturales}

Los factores socioculturales que determinan la utilización de los servicios de salud incluyen tecnología y valores. La tecnología es un factor sociocultural que a diferencia de los factores organizativos demuestra el relativamente bajo control que de ella tienen los administradores de

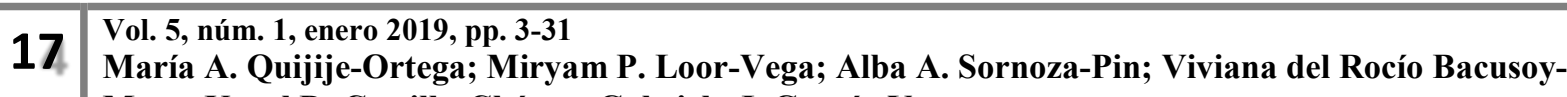
Mero; Karol D. Castillo-Chávez; Gabriela J. Garcés-Vera
} 
salud; ejerce influencia sobre la utilización de los servicios, en algunos baja el nivel de enfermedad o limita la necesidad de atención médica.

Los valores sociales también ejercen influencia sobre la utilización de los servicios de salud, teniendo en cuenta que el campo de los valores es un estudio relativamente difícil, dado que los valores, normas y creencias sociales afectan todos los otros aspectos y factores del proceso de atención médica.

\section{Factores de la Organización}

Esta segunda categoría de factores incluyen "las estructuras y procesos propios de la organización de la atención médica que rodean y afectan al proceso de atención médica (la interacción pacienteprofesional). Estos factores comprenden: la disponibilidad de recursos, la accesibilidad geográfica, la accesibilidad social, las características de la estructura (organización forma) y el proceso de prestación de servicios.

\section{Disponibilidad de recursos}

Se refiere a la relación entre el volumen y tipo de recursos existentes con los recursos necesarios para satisfacer las necesidades de atención sanitaria de la población, es decir la oferta de recursos. Se considera que un recurso está disponible cuando existe o es posible obtenerlo.

Generalmente, se evalúa la disponibilidad sobre una base geográfica relativamente amplia (por lo menos regional) y se expresa como volumen de recursos relativo a la población a la que están dirigidos.

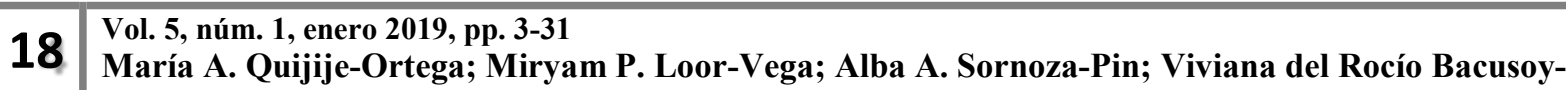
Mero; Karol D. Castillo-Chávez; Gabriela J. Garcés-Vera
} 
Accesibilidad geográfica

Se refiere a los factores espaciales que facilitan o entorpecen la utilización de recursos. Es la relación entre la ubicación de los recursos existentes y la ubicación de los usuarios (o de la necesidad). Este factor puede medirse en distancia, tiempo de viaje, o costos de viaje. La utilización de servicios de prevención depende más estrictamente de la accesibilidad geográfica que la utilización de servicios curativos, lo mismo ocurre con la asistencia a especialistas o la visita a médicos en comparación con la utilización de servicios públicos.

Cuanto más seria resulte la enfermedad o desorden y cuanto más sofisticado o especializado sea el recurso o servicio, menos importante o estrecha será la relación entre accesibilidad geográfica y volumen de servicios utilizados.

El concepto de accesibilidad temporal está estrechamente relacionado con la accesibilidad geográfica, Esta se refiere a las limitaciones sobre el momento en que se encuentra disponible (las horas que el médico atiende el consultorio, o el servicio ambulatorio permanece abierto, determinan la posibilidad de los usuarios, especialmente aquellos que trabajan, de recibir atención médica).

\section{Factores relativos a los profesionales}

Estos factores pueden dividirse en dos grupos:

Características económicas y características de la formación profesional.

Características económicas: Ha habido una creciente tendencia entre los economistas de salud en el sentido de que la interacción tradicional entre oferta y demanda no resulta aplicable en el mercado de la salud, por el contrario, la alternativa de "desplazamiento de la demanda" o hipótesis de la

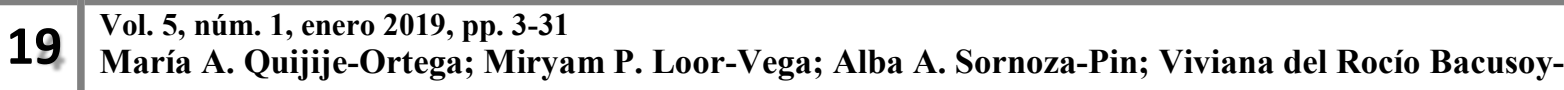
Mero; Karol D. Castillo-Chávez; Gabriela J. Garcés-Vera
} 
inducción, indica que los médicos tienen la capacidad de generar demanda para sus servicios. Algunos estudios revelaron que dicha hipótesis, la oferta, tecnología y número de médicos, parece tener decisiva importancia en la determinación de la utilización y gastos en servicios médicos.

Se llegó a algunas conclusiones: Los usuarios de los servicios no tienen verdadera conciencia de la magnitud de sus necesidades. La definición de necesidad es diferente según el punto de vista del profesional o del usuario. En muchos casos, no es el paciente quien toma la decisión sobre la atención a recibir.

Los usuarios a menudo no pueden evaluar que profesional puede ofrecer mejor servicio o que sustitutos se le pueden ofrecer. La aparición de una enfermedad en un momento dado es un fenómeno aleatorio, involuntario a menudo de carácter urgente, debido a esto y al hecho de que no sabe qué beneficios resultarán de la utilización de los servicios antes del tratamiento, los consumidores no pueden tomar decisiones "racionales" con respecto a éstos servicios. Los consumidores no saben a qué servicios recurrir, simplemente saben que desean ser tratados y dejan librada al médico la decisión de cuales servicios son adecuados.

\section{Perspectiva Epidemiológica}

Las poblaciones experimentan transformaciones demográficas, sociales, ambientales y económicas que influyen en su estado de salud. Existen condiciones de complejidad y variabilidad vinculadas a la actividad productiva del hombre que alteran el ambiente natural y social. Así las afecciones relacionadas con las condiciones ambientales y laborales coexisten con las enfermedades transmisibles.

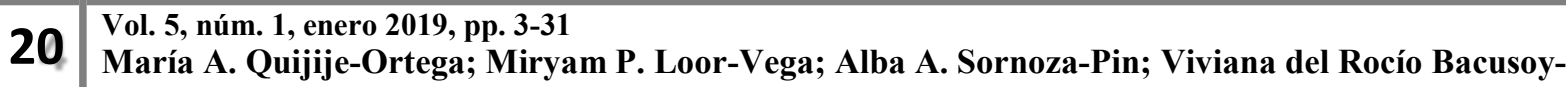
Mero; Karol D. Castillo-Chávez; Gabriela J. Garcés-Vera
} 
El comportamiento peculiar del ser humano entre todas las especies vivas, hace que, pese al progreso material, su vida se desarrolle entre la salud y la enfermedad. No es extraño, que el hospital sea una de las instituciones más solicitadas por las comunidades.

En este contexto, el profesional de salud necesita relacionar diversos factores para conocer las condiciones de salud de las poblaciones y una de las herramientas más importantes de que dispone es la epidemiología, cuya adecuada utilización puede ayudar a definir patrones de salud y enfermedades identificando los factores ambientales, de comportamiento y sociales que influyen en la salud de la comunidad, a la vez que brinda elementos objetivos para medir el impacto de las intervenciones. Para alcanzar la meta de salud para todos, el país amplió y reorganizó sus sistemas de salud, combinando a menudo programas y actividades para mejorar sus acciones en términos de equidad, eficacia y eficiencia.

Por un lado, el sector salud debe competir con otras esferas para obtener recursos financieros, en general extremadamente escasos. Por otro, grandes grupos de población tienen un acceso limitado a los servicios, con la consiguiente desprotección que ello significa. Es relevante, entonces, establecer un orden de prioridades, asignar recursos en base a las necesidades de salud de las poblaciones y valorar las repercusiones de los servicios.

\section{Perfiles de los servicios de emergencia (10)}

Los servicios de emergencia son importantes centros de manejo de enfermedades agudas y traumas, sin embargo, aparecen generalmente congestionados por un incremento en la demanda de pacientes. Por ello, varios estudios han tratado de determinar los factores que parecen influir en la demanda de los servicios de emergencia y sus asociaciones con variables socio demográficas.

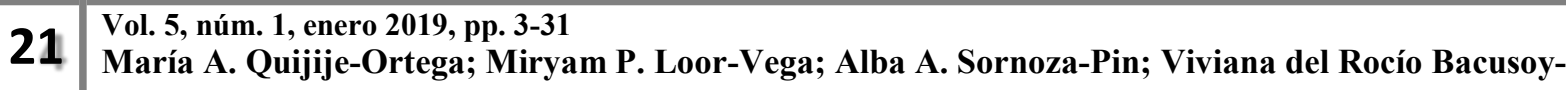
Mero; Karol D. Castillo-Chávez; Gabriela J. Garcés-Vera
} 
El Ecuador es un país plurinacional e intercultural. Se lo considera entonces, un país heterogéneo en grupos sociales incluso dentro de las diferentes regiones que lo conforman. Existe al momento 15,963,435 ecuatorianos (Datos INEC Marzo 2014), de los cuales 2,576,287 habitan en la provincia de Pichincha. Según el censo realizado en el año 2010, 68\% de la población se encuentra en el aérea urbana, predomina la población femenina (51.3\%) sobre la masculina (48.5\%). La edad promedio es de 29 años y el mayor porcentaje de la población está casada (40,1\%) en contraste con los solteros (36.9\%), aquellos que viven en unión libre (13.1\%), separados, viudos y divorciados $(3,7 \%, 3,2 \% 2,9 \%)$. La población en su mayoría se auto identifica mestiza $(82,1 \%)$ y el promedio de años de escolaridad, en personas de 24 años y más, es de 11,8 en los hombres y 11,1 en mujeres. De los 2,094,550 pichinchanos que están en edad de trabajar (personas de 10 años y más) apenas $1,249,950$ que equivale a 59, 17\% es económicamente activa, sin embargo 522,883 (47.55\%) no aportan al seguro social ni están afiliados a ningún otro tipo de seguro, que incluye seguro general, voluntario y campesino de IESS, ISSPOL e ISSFA, por lo que se deduciría que la población que utiliza los recursos de salud que ofrece el Ministerio de Salud Pública en esta provincia es significativo. (11)

\section{Usos de la Epidemiología (12)}

El desarrollo permanente del método epidemiológico y su cuerpo de conocimientos ha permitido diversificar sus usos y aplicaciones, algunos de los cuales se detallan a continuación:

\section{Medición del nivel de salud de poblaciones}

- Determinar la carga de enfermedad para planificar los requerimientos de servicios y la prioridad para la asignación de recursos.

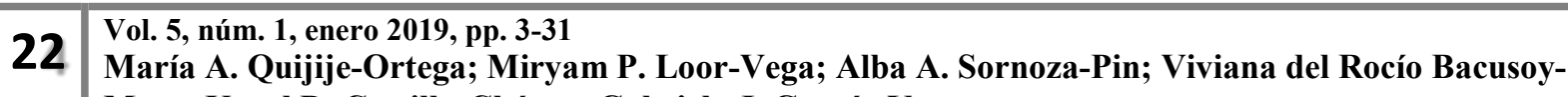
Mero; Karol D. Castillo-Chávez; Gabriela J. Garcés-Vera
} 
- Detección de tendencias en la incidencia o prevalencia de enfermedades.

- Identificación de cambios en los patrones de una enfermedad y sus consecuencias.

- Identificación de grupos de riesgo en la población.

- Determinación del estado de salud, la magnitud de capacidad o de la discapacidad.

2. Descripción de la historia natural de la enfermedad

- Definición de rangos de normalidad y/o valores esperados.

- Completar el cuadro clínico de una enfermedad e identificar condiciones predisponentes. Identificar extensión de periodos de etapa pre-sintomática.

- Ayudar en la predicción (pronóstico) en la mejoría clínica con y sin intervenciones.

3. Identificación de los determinantes de las enfermedades

Este objetivo de investigación busca establecer la relación entre determinantes y condiciones relacionadas con la salud. Esto debiera permitir distinguir entre:

- Asociaciones de dependencia estadística - entre dos o más eventos, características o variables. Estas asociaciones pueden o no estar en relación causal y,

- Determinantes, vale decir, factores que pueden producir cambios en las condiciones de salud. Estos son factores que tienen una relación causal directa con problemas de salud.

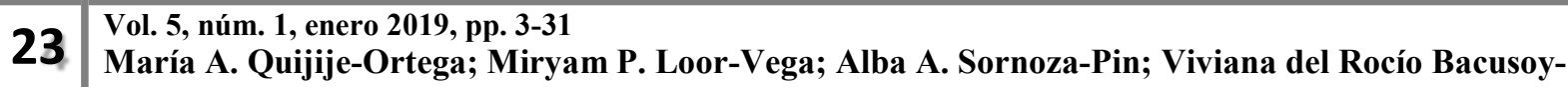
Mero; Karol D. Castillo-Chávez; Gabriela J. Garcés-Vera
} 
4. Control y prevención de la enfermedad

- Removiendo o eliminando agentes primarios, dependiendo del reservorio natural, modo de diseminación y sitio de acción

- Protección del ser humano mejorando las condiciones del medio (higiene) " Incrementando la resistencia del huésped (inmunización, incremento de la resistencia biológica)

- Modificación del comportamiento humano para impedir riesgos o promover acciones saludables.

5. En la selección de métodos de control y prevención:

- Identificando (estudios descriptivos), grupos de mayor riesgo.

- Identificando factores cuantitativamente importantes (epidemiología analítica)

- Métodos efectivos para el control y prevención (estudios experimentales).

6. Planificación $\quad \mathrm{y}$ evaluación $\quad$ de $\quad$ servicios $\quad$ de $\quad$ salud

En el pasado los servicios eran planificados y los recursos asignados sobre la base de la utilización histórica. La planificación lógica y la efectiva administración de los servicios de salud dependen de la estimación de las necesidades y de las demandas estimadas. Para esto se requiere información referente a:

- Identificación de los principales riesgos para la salud de la comunidad

- Conocimiento de la eficacia de las intervenciones

24 Vol. 5, núm. 1, enero 2019, pp. 3-31 
- $\quad$ Evaluación de la eficacia y efectividad de las intervenciones propuestas

7. Misceláneos

- Lectura crítica de información científica

- Predicción de escenarios sanitarios

- Estudio de la forma en que se distribuyen los recursos de acuerdo con las necesidades de la población

- Aplicación de sus métodos al escenario clínico.

El Ecuador como Estado Parte del Reglamento Sanitario Internacional, se orientaba a vigilar, informar, controlar y notificar cuando un área quedaba exenta de alguna de las siguientes seis enfermedades transmisibles: cólera, peste, fiebre amarilla, viruela, fiebre recurrente y tifus; de las cuales, sólo tres: cólera, peste o fiebre amarilla eran consideradas de gran potencial de propagación entre los países. En 1973, este instrumento fue modificado añadiéndose disposiciones relativas al Cólera, y en 1981 se lo revisó excluyéndose de la vigilancia a la viruela.

En el 2001, se creó la Red Andina de Vigilancia Epidemiológica (RAVE), en el marco de la iniciativa de la creación del Escudo Epidemiológico Andino, en el que participaba Ecuador y le confería, desde entonces, responsabilidad en la vigilancia prioritaria de: Cólera, Fiebre amarilla, Sarampión, Dengue clásico, Dengue hemorrágico, Malaria falciparum, Malaria vivax, VIH/SIDA, Tuberculosis, Enfermedad de Chagas y enfermedades relacionadas con la altura con especial atención a las zonas fronterizas. (13)

Desde el 2004, Ecuador participa en un esquema de vigilancia subregional consignado en el Plan Integrado de Desarrollo Social (PIDS), en lo que, concierne a la seguridad sanitaria subregional 
se concreta operativamente en los Proyectos: "Control de Malaria en las zonas fronterizas de la región Andina: Un enfoque comunitario PAMAFRO y el proyecto "Fortalecimiento del Plan Andino de Salud en Fronteras- PASAFRO”. En junio del 2007, en el ámbito mundial de salud, entra en rigor el Nuevo Reglamento Sanitario Internacional (RSI-2005), en el contexto de la vigilancia en el Ecuador, se circunscribe de manera vinculante al marco jurídico de este Reglamento, en que se establece un proceso de dos etapas para que la OMS, pueda ayudar a los Estados Partes a planificar el cumplimiento de sus obligaciones a este respecto. En el 2007, el Organismo Andino de Salud realizó una evaluación de la situación de la RAVE, que identificaba entre los principales aspectos que los objetivos definidos en su creación no eran pertinentes para la fecha, en consideración a la modificación del panorama epidemiológico, así como la constatación de una limitada coordinación entre las redes de vigilancia, básicamente la red del MERCOSUR, RAVE, Emergentes y Reemergentes, Cono Sur y Amazonía, entre otras. (14)

Particularmente importante, en el presente, es la adecuada realización de la reorganización de la Atención Primaria de Salud, la cual es la base de nuestro sistema y requiere de una profundización y empleo de los instrumentos técnicos que le permitan cumplir con su rol de una manera eficaz, efectiva y eficiente. El objetivo primordial en este nivel de atención es, partiendo de un correcto análisis de la situación de salud, realizar una buena dispensarización de la población a cargo de cada Equipo Básico de Salud, es imprescindible que dichos equipos utilicen bien el Enfoque de riesgo que no es otra cosa que un método que se emplea para medir la necesidad de atención por parte de grupos específicos. Ayuda a determinar prioridades de salud y es también una herramienta para definir las necesidades de reorganización de los servicios de salud. Intenta mejorar la atención para todos, pero prestando mayor atención a aquellos que más lo requieran. Es un enfoque no igualitario, discrimina a favor de quienes tienen mayor necesidad de atención. 
A partir de la correcta dispensarización el reorganizar el sistema para que responda a las reales necesidades de modificar el estado de salud de la población, con un enfoque más promocional y preventivo, será un enorme reto que sólo podremos enfrentar exitosamente con un profundo conocimiento técnico que permita liderar este proceso y en el cual todos los trabajadores de la salud debemos sentirnos profundamente comprometidos.

Los departamentos de emergencia de los hospitales son un componente fundamental e indispensable del sistema de atención de salud a nivel mundial. Si bien su misión tradicional es proporcionar servicios de trauma y emergencia para las personas en peligro inminente de perder la vida o sufrir daños permanentes a su salud, el papel de los servicios de urgencias se ha ampliado durante las últimas décadas. Los departamentos de emergencia están en la primera línea de los esfuerzos y las respuestas a los desastres naturales, otros eventos con víctimas en masa y emergencias de salud pública derivados de los brotes y epidemias de gripe (influenza) y otras enfermedades transmisibles de gran propagación

El epidemiólogo de área debe mantener comunicación eficiente y Oportuna con el registro civil para conocer de inmediato las defunciones con certificación médica por enfermedades de importancia epidemiológica.

El conocimiento de alguna defunción con estas características debe significar para él la inmediata investigación retrospectiva para su confirmación o descarte. No debe olvidarse que, aun con un buen índice de notificación, pueden pasar desapercibidos casos y defunciones por enfermedades cuarentenables y de acción sanitaria. Se requiere aquí también obtener datos más completos y exactos.

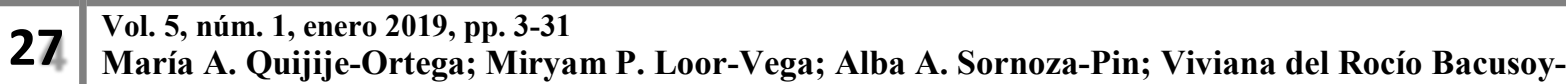
Mero; Karol D. Castillo-Chávez; Gabriela J. Garcés-Vera
} 
El epidemiólogo debe tender constantemente a mejorar la calidad de los registros de mortalidad. El estudio y correlación constante de los índices de morbilidad y mortalidad es un medio útil para juzgar la bondad de los sistemas estadísticos correspondientes. Situación epizootiológica Es muy importante la vigilancia epidemiológica cuando se trata de epizootias transmisibles al hombre, como es el caso de la encefalomielitis equina, la tuberculosis en el ganado, la brucelosis, etc. Investigaciones de laboratorio Las investigaciones de laboratorio pueden referirse a hallazgos relacionados con el agente (aislamiento de cepas o características especiales de ellas) o al estado inmunitario de la comunidad contra determinada afección.

El epidemiólogo debe procurar el aislamiento, identificación y estudio de los agentes causales de enfermedad, así como colaborar con la asesoría de epidemiología de nivel nacional en la realización de encuestas serológicas periódicas de la comunidad, mediante muestras estadísticamente representativas, por grupos de edad.

Los resultados de estos estudios, unidos a los otros datos comentados antes, son decisivos para planificar los programas de vacunación, de tal manera que cubran las zonas geográficas y los grupos de población que realmente los necesitan, así como para evaluar después su impacto en el estado inmunitario de esta población vacunada. La organización de los servicios de epidemiología a nivel local, dentro del área de salud, y su supervisión y evaluación constantes constituyen función primordial del epidemiólogo de área; en este sentido, su relación personal con otros epidemiólogos y con los funcionarios que realizan las acciones propiamente tales es muy importante para el adecuado desarrollo de los programas. El epidemiólogo de área debe actuar además como asesor de los otros programas de salud del área, sugiriendo bases de evaluación de estos mediante la aplicación del 
método epidemiológico, con miras a conseguir que la ejecución de estos otros programas signifique la utilización efectiva de los recursos disponibles.

\section{Conclusiones.}

La salud es un derecho fundamental de las personas, para cuya consecución se necesita la colaboración y coordinación de distintos sectores técnicos y sociales. Entre ellos, el sistema de salud cumple un papel relevante, pero ha de contar siempre con los aportes de sectores como la educación, vivienda, comunicación, agricultura y ambiente, entre otros.

No existe un conjunto de datos que pueda responder a todas las necesidades de la epidemiología, pero si es posible definir un núcleo de datos a utilizar. La información debe estar dirigida a la acción y el hecho de que sea necesaria para planificar y realizar la gestión no justifica la obtención de ingente cantidad de datos. La disponibilidad de datos es una condición necesaria pero no suficiente para una buena planificación.

En consecuencia, un análisis epidemiológico en un modelo de información sanitaria responde a la necesidad de establecer un sistema de evaluación del estado de salud de la población y de las actividades de promoción, prevención y asistencia sanitaria. Un enfoque epidemiológico de la salud intenta abordar los problemas desde una perspectiva más amplia y representa un cambio cualitativo importante, que permite orientar las acciones en términos individuales, comunitarios y ambientales.

La epidemiología es una disciplina capaz de enriquecer la comprensión del mundo real en el que vivimos, fue descripta como el arte liberal y puede ser el instrumento de la justicia social, ya que constituye un elemento esencial en la planificación de la salud y la toma de decisiones en el 
objetivo de salud para todos. La epidemiología es parte esencial de la salud pública y suele ser dificultoso separar al epidemiólogo de los niveles de decisión; sin embargo, es posible y probablemente necesario separa las funciones del político de las de investigación y practica epidemiológica.

El nivel decisorio establece la selección. La política en salud se basa en la selección de problemas prioritarios en una comunidad determinada: elige los objetivos, los grupos de en los cuales habrá de trabajar, y las intervenciones y recursos necesarios. La elección de las intervenciones requiere la adecuada evidencia epidemiológica de la efectividad de los métodos propuestos, y constituye en último término una decisión política.

\section{Bibliografía.}

1. Ortegón E, Pacheco JF, Prieto A. Metodología del marco lógico para la planificación, el seguimiento y la evaluación de proyectos y programas Santiago de Chile: Naciones Unidas; 2005.

2. Macedo M. Infecciones Hospitalarias Montevideo: Universidad de la Republica; 2008.

3. González Rendón MG, Vásquez Chirinos MdlG. ENTIDADES NOSOCOMIALES PRESENTES EN EL SERVICIO DE TERAPIA INTENSIVA DEL HOSPITAL VARGAS DE CARACAS. Trabajo Especial de Grado que se presenta para optar al título de Especialista en Medicina Interna. Caracas: UNIVERSIDAD CENTRAL DE VENEZUELA, FACULTAD DE MEDICINA; 2015.

4. Hernández-Aguado I, Lumbreras B, Jarrín I. La epidemiología en la salud pública del futuro. Revista Española de Salud Pública. 2006 Oct; 80(5): p. 469-474.

5. Valdivia Onega NC. Epidemiología y atención primaria de salud. Revista Cubana de Higiene y Epidemiología. 2003 Dic; 41(2-3).

6. Larios Ortiz L, Hernández González M, Marrero Puig LG. Vinculación del residente de Higiene y Epidemiología a actividades epidemiológicas emergentes. Humanidades Médicas. 2015 Ago; 15(2): p. 215-220.

7. Leal de Valor D, Bolívar de Muñoz M. La Planificación Estratégica como proceso de 
integración de un equipo de salud. Enfermería Global. 2011 Oct; 10(24): p. 180-188.

8. Ase I, Burijovich J. La estrategia de Atención Primaria de la Salud:¿progresividad o regresividad en el derecho a la salud? Salud colectiva. 2009 Abr; 5(1): p. 27-47.

9. Linares Pérez N. Aplicación de los enfoques de salud de la población y los determinantes sociales en Cuba. Revista Cubana de Salud Pública. 2015 Mar; 41(1): p. 94-114.

10. Toranzo T, Aramburu F. Los profesionales de urgencias y emergencias: perfil actual y posibilidades de desarrollo. Anales del Sistema Sanitario de Navarra. 2010 Ene; 33(1): p. 13-19.

11. Ayala Mora E. Interculturalidad en el Ecuador Universidad Andina Simón Bolívar: Quito; 2008.

12. Alarcón J. Epidemiología: concepto, usos y perspectivas. Revista Peruana de Epidemiología. 2009 Abr; 13(1): p. 1-3.

13. MSP. Sistema Integrado de Vigilancia Epidemiológica Norma técnica Quito: Ministerio de Salud Pública del Ecuador; 2014.

14. MSP. NORMAS DEL SISTEMA INTEGRADO DE VIGILANCIA EPIDEMIOLÓGICA Quito: Ministerio de Salud Pública; 2013. 